\title{
A TEM Analysis of Particulates in a Polar Ice Core
}

\author{
E. V. Esquivel and L E. Murr \\ Department of Metallurgical and Materials Engineering, The University of Texas at El Paso, El \\ Paso, TX 79968
}

It is already well established that polar and glacier ice cores can provide a global historical or prehistorical perspective for atmospheric and global climatic features and events. Heavy particulate banding in deep cores $(2-3 \mathrm{~km})$ from Antarctica have indicated volcanic catastrophes dating to 150,000 years ago while air trapped in bubbles in the ice has provided a record of atmospheric methane and other gases in antiquity [1]. Gas analyses in particular have provided a context for human-related contributions to the global atmosphere over the centuries [1]. Recent studies also point to the increasingly toxic effects of ultra-fine/nanoparticles suspended in the ambient air [2,3], and the fact that these particles are mostly agglomerates of crystalline material $[4,5]$.

This paper explores the prospects for analyzing fine and ultra-fine particulates in ice utilizing transmission electron microscopy (TEM) and associated analytical methods, including selected-area electron diffraction (SAED) and elemental analysis by energy dispersive (X-ray) spectrometry (EDS). The technique involves careful extraction of an ice core sample in a clean-room environment and allowing the core sample to melt in a clean container. Drops ( $\sim 2 \mathrm{~mm}$ diameter $)$ from random samples pipetted from the ice melt were transferred to carbon/formvar-coated TEM grids [6] and allowed to dry down (protected from the air), and were then observed in the TEM.

Figure 1 provides some typical examples of the TEM analysis of drops from an ice core sample from the Greenland ice cap, corresponding to roughly 10,000 years ago. Figure 1(a) and (b) show a complex silicate aggregate composed of overlapping nanocrystals. Similar, complex, microcrystalline aggregates have been observed in current ambient air [4,5]. Figure 1(c) and (d) show a crystalline, mineral aggregate composed of $\mathrm{Si}, \mathrm{Al}, \mathrm{Fe}$, and $\mathrm{O}$. Figure 1 illustrates the ability to utilize TEM analysis to fully characterize very tiny particulates which have been collected on TEM support grids. It is interesting to note that the lungs of the 5300 year old Stone Age hunter found in the ice of the alpine glaciers exhibited a profusion of nanoparticles, mostly attributed to the combustion of organic matter [7]. The lung tissue contained anthracotic areas consisting of silicates as in Fig. 1, and carbonaceous particulates. $\mathrm{TiO}_{2}$ nanocrystals were also observed [8].

\section{References}

[1] R. A. Rasmussen and M. A.K. Khalil, J. Geophysical Res. 89 (1984) 11, 599.

[2] L. C. Renwick et al., Toxicol. Appl. Pharmacol. 172 (2) (2001) 119.

[3] Q. Zhiqiang et al., Atmos. Environ. 34 (2000) 443.

[4] J. J. Bang and L. E. Murr, J. Mater. Sci. Letters 21 (2002) 361.

[5] J. J. Bang and L., E. Murr, JOM 54 (12) (2002) 28.

[6] L. E. Murr and K. Kloska, Water Res. 10 (1976) 469.

[7] F. Hofer et al., Spektrum der Wiss. Oct. (1998) 48.

[8] Research supported in part by a Murchison Endowment, TX Tobacco Settlement funds, and an EPA Grant (Project No. A-02-5) through the Southwest Center for Environmental Research and Policy (SCERP). R.A. Rasmussen of the Oregon Graduate Institute supplied the ice core sample. 


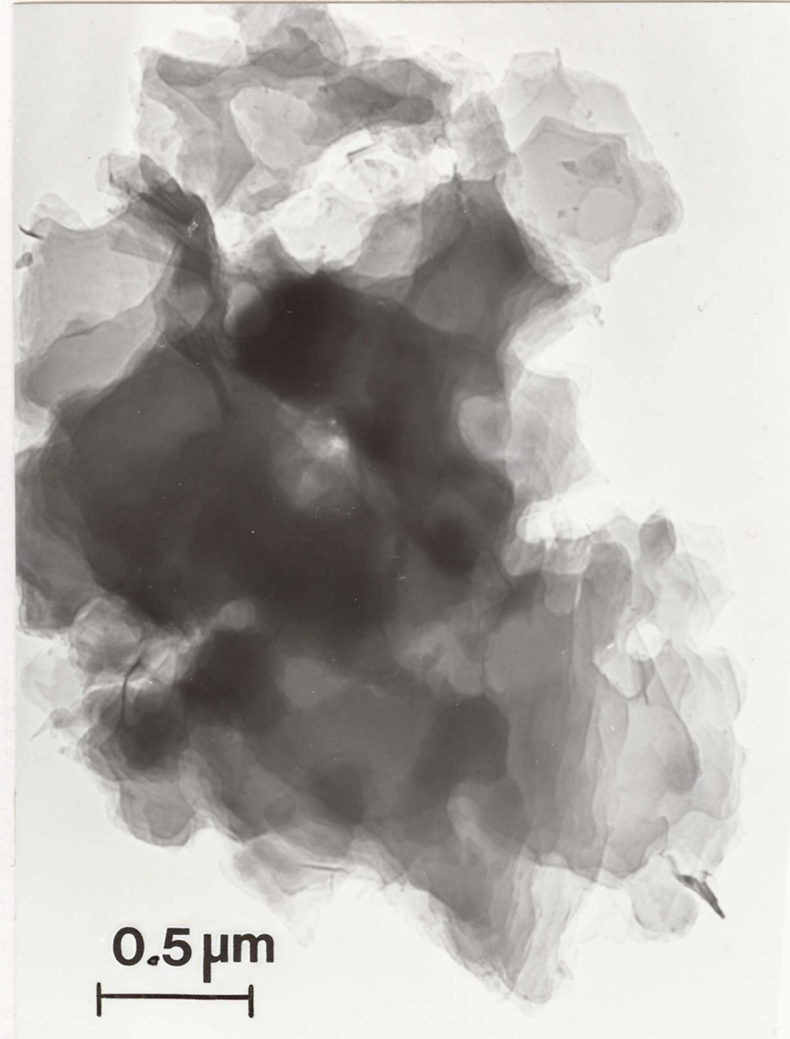

a

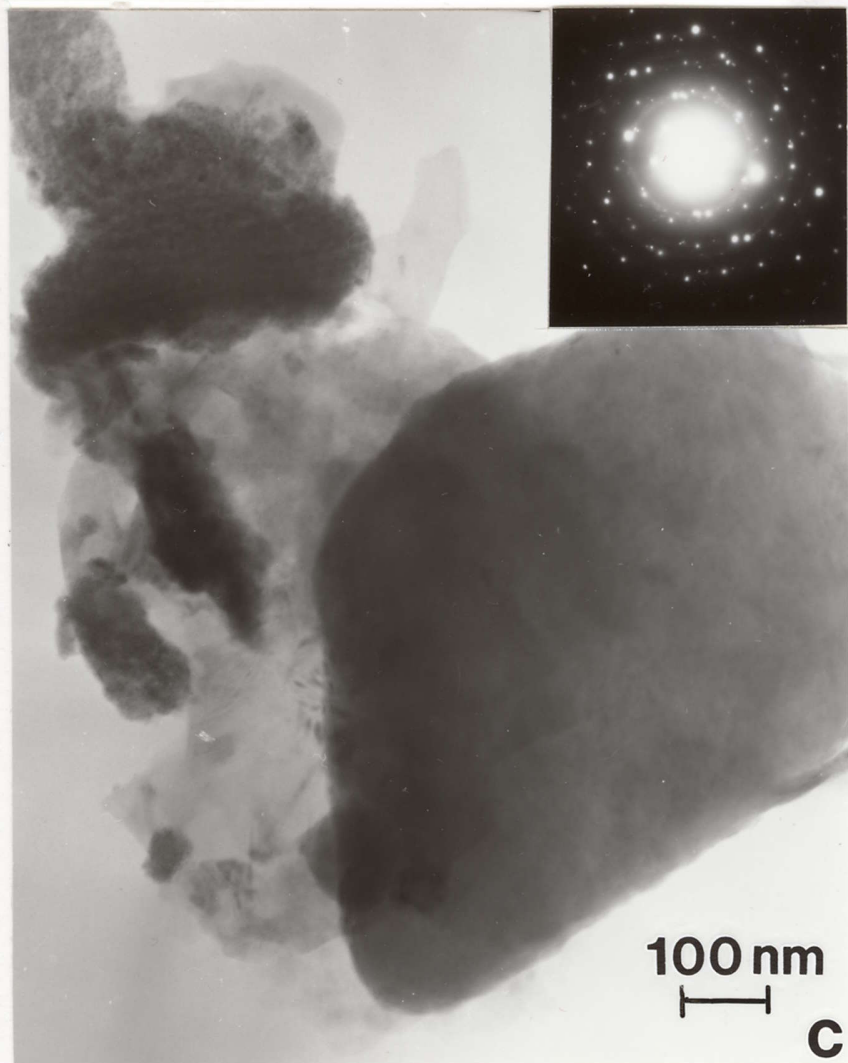

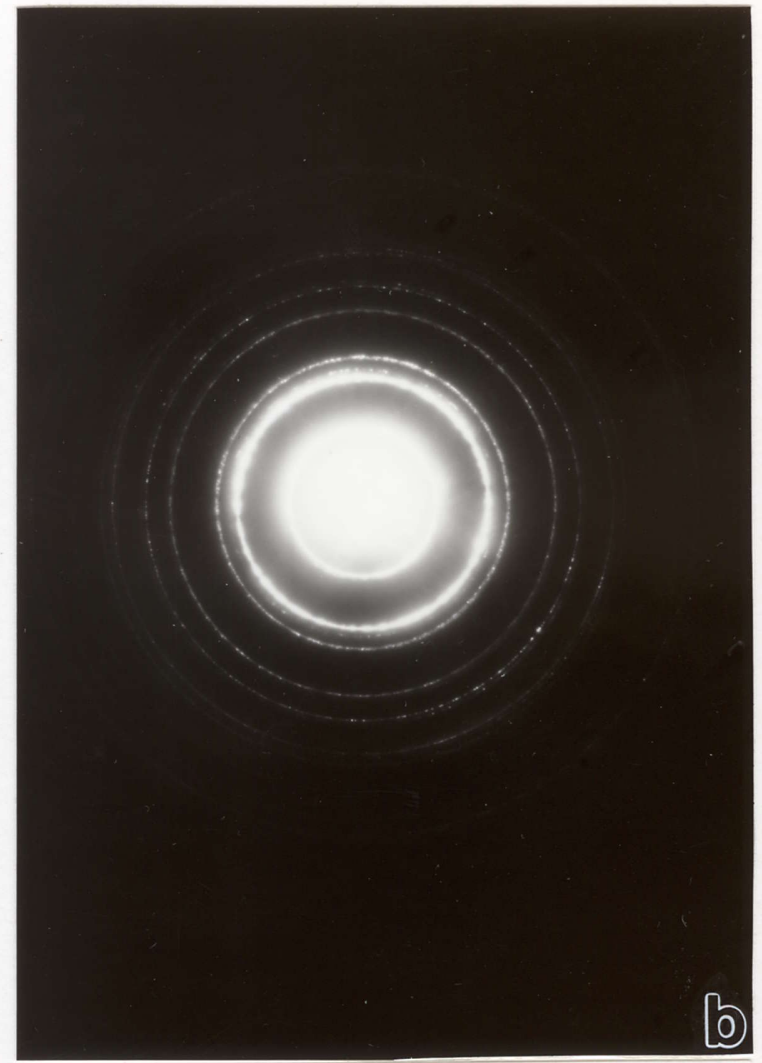

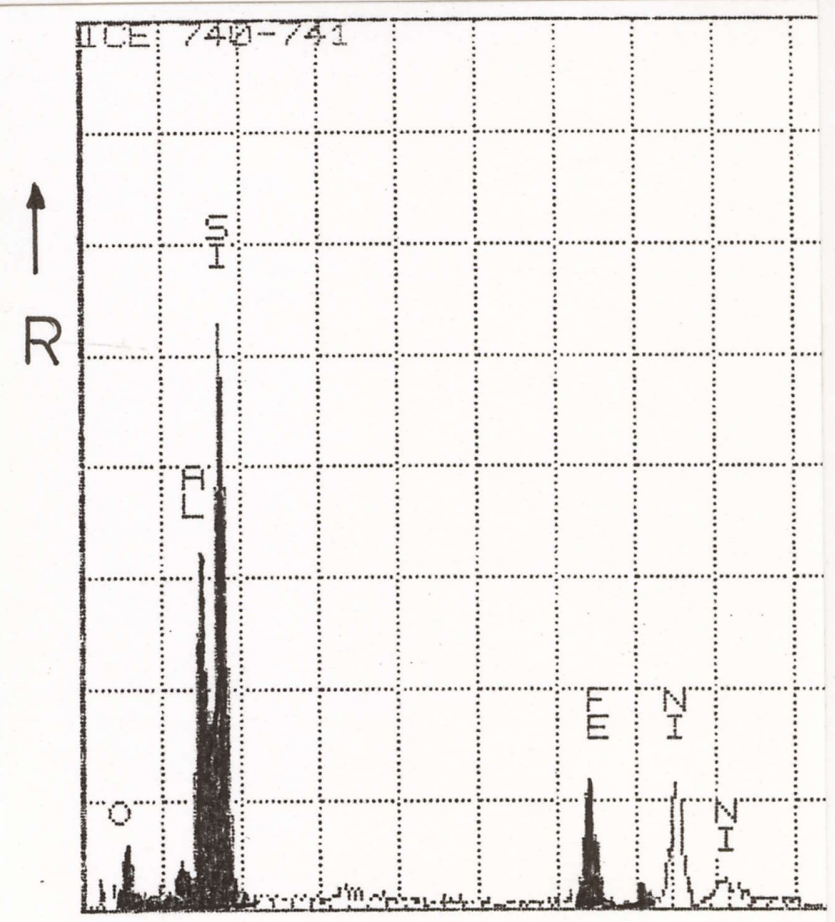

O

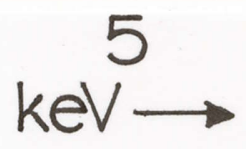

FIG. 1. (a) and (b) show a complex polycrystalline, silicate agglomerate. (a) is a bright-field TEM image and (b) is the corresponding SAED pattern. (c) and (d) show an aluminum-iron-silicate agglomerate of large crystals as indicted by the SAED pattern insert in (c). 